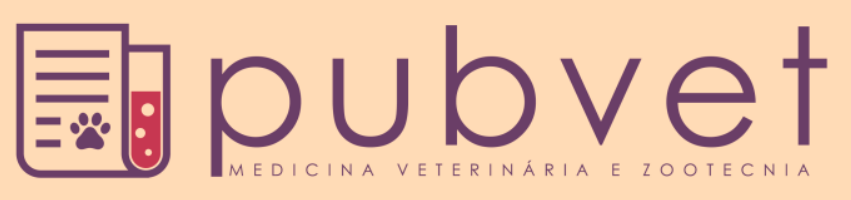

https://doi.org/10.31533/pubvet.v12n11a218.1-5

\title{
Simulação do efeito sedativo de doses altas de detomidina em equinos
}

\author{
Lucas Valeiras Gaddini ${ }^{1}$, Marilda Onghero Taffarel $^{2} \bullet$, Marcos Ferrante $^{2 *} \bullet$ \\ ${ }^{I}$ Mestrando do programa de Pós-Graduação em Produção Sustentável e Saúde Animal, Universidade Estadual de Maringá, Umuarama, PR Brasil. \\ ${ }^{2}$ Docente do curso de Medicina Veterinária da Universidade Estadual de Maringá, Umuarama, PR Brasil. \\ * Autor para correspondência: marcosferrante@ gmail.com
}

\begin{abstract}
RESUMO. O objetivo do presente estudo foi simular o efeito sedativo da administração de detomidina nas doses de $80 \mathrm{ug} / \mathrm{kg}$ e $30 \mathrm{ug} / \mathrm{kg}$ por via intravenosa e intramuscular em equinos. Foi realizado um modelamento farmacocinético/farmacodinâmico (PK/PD) com a curva sigmoide de concentração plasmática, obtidas por Mama e colaboradores, em função do efeito na posição da cabeça mediante a equação de Hill. Seguidamente foi realizada uma simulação do efeito sedativo das doses de $80 \mathrm{ug} / \mathrm{kg}$ via intravenosa (IV) e intramuscular (IM) por meio da equação de Hill. As concentrações plasmáticas maiores e mais prolongadas obtidas após as administrações de $80 \mathrm{ug} / \mathrm{kg}$, quando comparadas às administrações de $30 \mathrm{ug} / \mathrm{kg}$ resultaram em efeitos superior e mais duradouros. A simulação do efeito de detomidina após a administração de $80 \mathrm{ug} / \mathrm{kg}$ via IV determinou efeitos na posição da cabeça superiores a $90 \%, 75 \%$ e $50 \%$ até 2,4 e 6 horas após administração. Enquanto que a simulação do efeito de detomidina após a administração de $80 \mathrm{ug} / \mathrm{kg}$ via IM determinou efeitos na posição da cabeça superiores a 90\%, 75\% e 50\% até 3, 4 e 6 horas após administração. A presente simulação demostrou que os efeitos da posição da cabeça após a administração de $80 \mathrm{ug} / \mathrm{kg}$ de detomidina por via IV e IM em equinos são superiores e mais duradouros que administrações de $30 \mathrm{ug} / \mathrm{kg}$. Todavia, estudos in vivo devem ser realizados a fim de verificar os potenciais efeitos adversos dessas dosagens em equinos.
\end{abstract}

Palavras chave: Modelamento farmacocinético/farmacodinâmico, alfa 2 agonistas, farmacologia clínica

\section{Simulation of the sedative effect of high doses of detomidine in equines}

ABSTRACT. The objective of the study was to simulate the sedative effect of the
administration of detomidine in doses of $80 \mathrm{ug} / \mathrm{kg}$ by intravenous and intramuscular route
in equines. A pharmacokinetic/pharmacodynamic (PK/PD) model was performed with the
sigmoid curve of plasma concentration, obtained by Mama and collaborators, depending
on the effect on the head position by means of Hill equation. A sedative effect simulation
of $80 \mathrm{ug} / \mathrm{kg}$ intravenous (IV) and intramuscular (IM) doses by Hill's equation was then
performed. Higher and prolonged plasmatic concentrations were obtained after
administrations of $80 \mathrm{ug} / \mathrm{kg}$, when compared to administrations of $30 \mathrm{ug} / \mathrm{kg}$ resulting in
higher and longer effect. The simulation of the effect of detomidine after administration of
80 ug $/ \mathrm{kg}$ IV determined effects in the head height higher than $90 \%, 75 \%$ and $50 \%$ up to 2 ,
4 and 6 hours after administration. Meanwhile, the simulation of the effect of detomidine
after the administration of $80 \mathrm{ug} / \mathrm{kg}$ IM determined effects in head height higher than $90 \%$,
$75 \%$ and $50 \%$ up to 3,4 and 6 hours after administration. The present simulation showed
that the effects of the position of the head after the administration of 80 ug $/ \mathrm{kg}$ of detomidine
via IV and IM in equines are superior and more prolonged when compared to the
administration of $30 \mathrm{ug} / \mathrm{kg}$. Therefore, in vivo studies should be performed in order to
verify the adverse effects of these dosages in equines.

Keywords: Pharmacokinetic/pharmacodynamic modeling, alpha 2 agonists, clinical pharmacology 


\title{
Simulación del efecto sedante de dosis altas de detomidina en equinos
}

\begin{abstract}
RESUMEN. El objetivo del estudio fue simular el efecto sedante de la administración de detomidina a dosis de $80 \mathrm{ug} / \mathrm{kg}$ por vía intravenosa e intramuscular en equinos. Se realizó un modelado fármaco cinético/fármaco dinámico (PK/PD) con la curva sigmoide de concentración plasmática obtenidas por Mama y colaboradores, en función del efecto em la posición de la cabeza mediante la ecuación de Hill. A continuación se realizó una simulación del efecto sedante de las dosis de $80 \mathrm{ug} / \mathrm{kg}$ por vía intravenosa (IV) e intramuscular (IM) por medio de la ecuación de Hill. Las concentraciones plasmáticas mayores y más prolongadas obtenidas después de las administraciones de $80 \mathrm{ug} / \mathrm{kg}$ respecto de las administraciones de $30 \mathrm{ug} / \mathrm{kg}$ resultaron en efectos superiores y más duraderos. La simulación del efecto de detomidina después de la administración de $80 \mathrm{ug} / \mathrm{kg}$ vía IV determino efectos em la posición de la cabeza superior al 90\%, 75\% y 50\% hasta 2 , 4 y 6 horas después de la administración. Mientras que la simulación del efecto de detomidina después de la administración de $80 \mathrm{ug} / \mathrm{kg}$ vía IM determino efectos em la posición de la cabeza superiores al 90\%, 75\% y 50\% hasta 3, 4 y 6 horas después de la administración. La presente simulación demuestra que los efectos de la posición de la cabeza tras las administraciones de $80 \mathrm{ug} / \mathrm{kg}$ de detomidina por vía IV e IM en equinos son superiores y más dorados respecto de Las administraciones de $30 \mathrm{ug} / \mathrm{kg}$. Sin embargo, los estudios in vivo debían realizarse con el fin de ver los posibles efectos adversos de estas dosis en equinos.
\end{abstract}

Palabras clave: Modelado farmacocinético/farmacodinámico, alfa 2 agonistas, farmacología clínica

\section{Introdução}

Sedativos e analgésicos têm sido amplamente utilizados para procedimentos diagnósticos e pequenos procedimentos cirúrgicos em equinos em estação (LeBlanc, 1991). Uma sedação ideal é descrita como uma propriedade em que o paciente se encontra sonolento, respondendo a estímulos ambientais, sem riscos e movimentos excessivos (Motta et al., 2016). Cloridrato de detomidina é um agonista $\alpha-2$ comumente utilizado na sedação, analgesia visceral ou como medicação préanestésica em cavalos, e isso é devido ao efeito do fármaco no sistema nervoso central (Virtanen, $\underline{1986)}$.

O conhecimento da concentração plasmática necessária para o efeito desejado é o passo inicial para estimar seu efeito (Jones et al., 2012). A compreensão da farmacocinética (PK) permite o ajuste de dosagens a fim de controlar a concentração no local de efeito, assim como compreender a farmacodinâmica (PD) ajuda a titular o fornecimento de anestésico de acordo com as necessidades individuais e sucessivas do paciente (Billard, 2015). Entender e modelar PK / PD em uma espécie pode permitir que sejam feitas predições com outras doses (Whiteside et al., 2013). Baseia-se em estabelecer a relação que existem entre a concentração de fármaco no plasma e os efeitos causados a partir desta (Billard, $\underline{2015)}$.
Estudos realizados por Mama et al., (2009) determinaram as concentrações plasmáticas e os efeitos sedativos após doses de $30 \mathrm{ug} / \mathrm{kg}$ pelas vias intravenosa e intramuscular em equinos. Enquanto que, Salonen et al. (1989) determinaram as concentrações plasmáticas após quando administradas doses de $80 \mathrm{ug} / \mathrm{kg}$ pela via intravenosa e intramuscular em equinos, porem não foram estabelecidos os efeitos sedativos destas altas doses. O objetivo do presente estudo foi simular o efeito sedativo da administração de detomidina na dose de $80 \mathrm{ug} / \mathrm{kg}$ por via intravenosa e intramuscular em equinos.

\section{Material e métodos}

Foi realizado um modelamento PK/PD com a curva sigmoide de concentração em função do efeito (Figura 1) mediante a equação de Hill descrita na figura 2 (Billard, 2015). O efeito sedativo foi considerado a partir da posição da cabeça medida em centímetros desde o ponto mais ventral do queixo até o chão. Os dados da concentração plasmática de detomidina e do efeito na posição da cabeça correspondentes foram obtidos do estudo realizado por Mama et al. (2009) após a administração de $30 \mathrm{ug} / \mathrm{kg}$ pelas vias intravenosa e intramuscular em equinos. Para a simulação do efeito sedativo das doses de $80 \mathrm{ug} / \mathrm{kg}$ pelas vias intravenosa e intramuscular foram utilizados os dados do estudo de farmacocinética realizado por Solonen et al. (1989). A simulação 
do efeito foi realiza estimando os valores do efeito correspondentes a cada concentração plasmática por médio da equação de Hill.

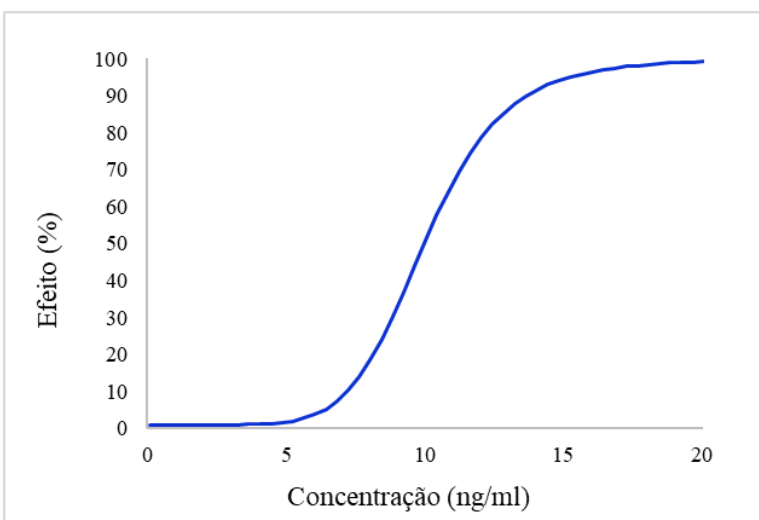

Figura 1. Curva sigmoide de concentração em função do efeito (Adaptado de Billard, et al., 2015).

$$
\mathrm{E}=\mathrm{E}_{0}+\mathrm{E}_{\max } \mathrm{x} \frac{\mathrm{C}^{\gamma}}{\mathrm{C} 50^{\gamma}+\mathrm{C}^{\gamma}}
$$

Figura 2. Equação de Hill. Onde "E" indica o efeito estimado, "E0" o efeito inicial, " $\mathrm{E}_{\max } \mathrm{x}$ " o efeito máximo, "C" a concentração, "C50" a concentração para o $50 \%$ do efeito e " $\gamma$ " o coeficiente de Hill.

\section{Resultados e discussão}

Love et al. (2015) mensuraram a farmacocinética da buprenorfina a uma dose em bolus de $1 \mu \mathrm{g} / \mathrm{kg}$ com aplicação intravenosa (IV) em equinos. Juntamente estímulos nociceptivos, térmico e mecânico, parâmetros fisiológicos e função locomotora foram analisados e comparados com dados também coletados de um grupo de tratamento controle (Love et al., 2012). $\mathrm{O}$ modelamento PK/PD a partir dos dados coletados permitiu realizar simulações dos efeitos com diferentes doses utilizando parâmetros de média aritmética PD (contagem de passos), parâmetros de média geométrica PK ou parâmetros PD (limiar mecânico). Dessa maneira seu estudo apoia o uso da buprenorfina, pela adequação de dose realizada, para analgesia perioperatória (Love et al., 2015).

De modo equivalente, no presente estudo de simulação, foram selecionados os dados da concentração plasmática de detomidina e do efeito na posição da cabeça após a administração de 30 $\mathrm{ug} / \mathrm{kg}$ obtidos por Mama et al. (2009), para simular os efeitos consequentes pelas concentrações plasmáticas à administrações nas doses de 80 $\mathrm{ug} / \mathrm{kg}$, pelas mesmas vias de administração (Solonen et al., 1989). Foi escolhido o efeito da posição da cabeça por ser um parâmetro que permite medir objetivamente o nível de sedação (Mama et al., 2009).

$\mathrm{Na}$ figura 3 se apresentam as curvas da farmacocinética após a administração de detomidina nas doses de $30 \mathrm{ug} / \mathrm{kg}$ e $80 \mathrm{ug} / \mathrm{kg}$ nas vias intramuscular e intravenosa em equinos. $\mathrm{O}$ modelamento PK/PD realizado mediante a equação de Hill com a concentração plasmática de detomidina em função do efeito na posição da cabeça em equinos se apresenta na figura 4. O presente estudo permitiu simular os efeitos da posição da cabeça em função da concentração plasmática observáveis após as administrações de $80 \mathrm{ug} / \mathrm{kg}$ de detomidina por via IV e IM em equinos (Figuras $5 \underline{\mathrm{e}}$ 6).

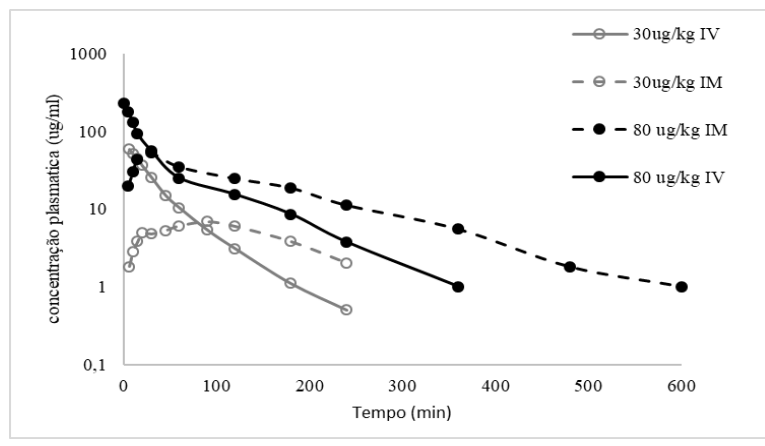

Figura 3. Farmacocinética plasmática da administração de detomidina nas doses de $30 \mathrm{ug} / \mathrm{kg}$ e $80 \mathrm{ug} / \mathrm{kg}$ nas vias intramuscular e intravenosa em equinos. (Adaptado de Mama et al,. 2009 e Solonen et al.,1989).

Todos os $\alpha 2$-agonista produzem efeitos confiáveis quanto a analgesia, sedativo e efeito miorelaxante (Rohbach et al., 2009), e são caracterizados por um efeito "teto" de sedação, pelo qual o aumento da dose prolonga a duração, mas não aumenta a intensidade da sedação (Valverde, 2010). A simulação do presente estudo permitiu determinar que as concentrações plasmáticas maiores e mais prolongadas obtidas após as administrações de $80 \mathrm{ug} / \mathrm{kg}$, a respeito das administrações de $30 \mathrm{ug} / \mathrm{kg}$, resultaram em efeitos superiores e mais duradouros (Figuras $3, \underline{5}$ e $\underline{6}$ ).

A simulação do efeito de detomidina após a administração de $80 \mathrm{ug} / \mathrm{kg}$ via IV determinou efeitos na posição da cabeça superiores a $90 \%$, $75 \%$ e $50 \%$ até 2,4 e 6 horas após administração (Figura 5). Enquanto que a simulação do efeito de detomidina após a administração de $80 \mathrm{ug} / \mathrm{kg}$ via IM determinou efeitos na posição da cabeça superiores a 90\%, $75 \%$ e $50 \%$ até, respectivamente, 3 , 4 e 6 horas após administração (Figura 6). 


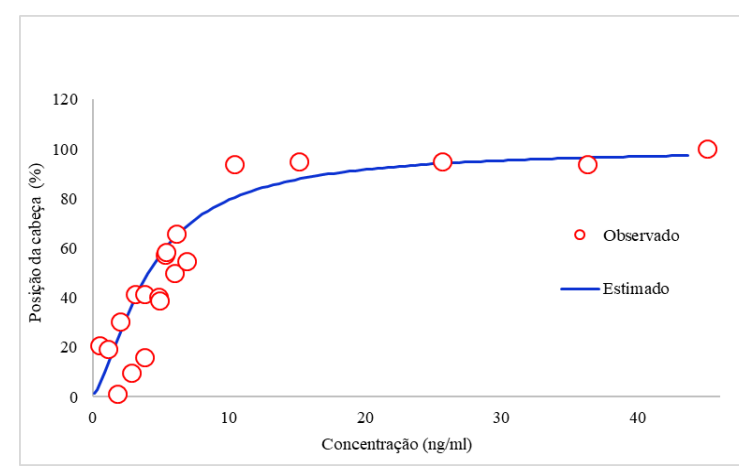

Figura 4. Modelamento farmacocinético/farmacodinâmico da concentração plasmática de detomidina em função do efeito na posição da cabeça em equinos realizado mediante equação de Hill.

A Cmax média de detomidina após a dose de $80 \mathrm{ug} / \mathrm{kg}$ via IM foi de $51,3 \mathrm{ng} / \mathrm{ml}$ foi menor que a Cmax media após a dose de $30 \mathrm{ug} / \mathrm{kg}$ por via IV $(104,5 \mathrm{ng} / \mathrm{ml})$ (Mama et al., 2009) (Figura 3). Isso sugere que a doses de $80 \mathrm{ug} / \mathrm{kg}$ via IM não determinaria efeitos farmacológicos superiores a doses de $30 \mathrm{ug} / \mathrm{kg}$ por via IV. No entanto, a dose de $80 \mathrm{ug} / \mathrm{kg}$ por via IV, que atinge Cmax de 231,7ng/ml (Salonen, 1989), poderia determinar efeitos farmacológicos superiores aos observados após administração da dose de $30 \mathrm{ug} / \mathrm{kg}$ por via IV.

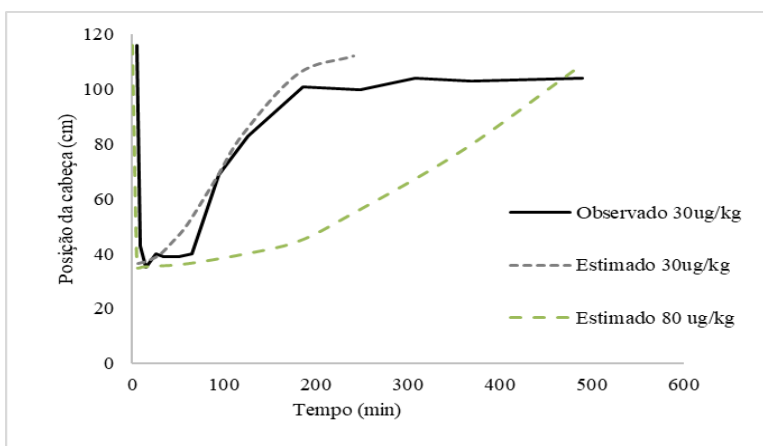

Figura 5. Efeito observado e estimado da posição da cabeça de equinos após administração das doses de $30 \mathrm{ug} / \mathrm{kg}$ e 80 $\mathrm{ug} / \mathrm{kg}$ de detomidina por via intravenosa em equinos.

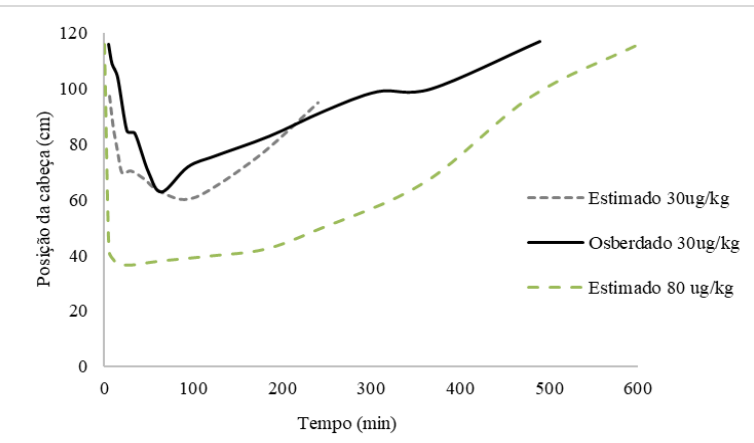

Figura 6. Efeito observado e estimado na posição da cabeça de equinos após administração das doses de $30 \mathrm{ug} / \mathrm{kg}$ e 80 $\mathrm{ug} / \mathrm{kg}$ de detomidina por via intramuscular em equinos

Agonistas alfa-2, como a xilazina, detomidina, medetomidina e dexmedetomidina são analgésicos e sedativos eficazes, porem também produzem alterações fisiológicas como bradicardia, hipertensão, bloqueio atrioventricular e ataxia (Valverde, 2010). Portanto, devem ser realizados estudos in vivo a fim de verificar os potenciais efeitos adversos das doses de $80 \mathrm{ug} / \mathrm{kg}$ via IV e IM em equinos os quais podem potencialmente limitar seu uso em alguns casos clínicos.

\section{Conclusão}

A presente simulação demostrou que os efeitos da posição da cabeça após a administrações de 80 $\mathrm{ug} / \mathrm{kg}$ de detomidina por via IV e IM em equinos são superiores e mais duradouros respeito das administrações de $30 \mathrm{ug} / \mathrm{kg}$. Porem, estudos in vivo devem ser realizados a fim de verificar os potenciais efeitos adversos dessas dosagens em equinos.

\section{Referências}

Billard, V. 2015. Pharmacokineticpharmacodynamic relationship of anesthetic drugs: from modeling to clinical use. F1000 research, France, 4(5):1-10.

Jones, H. M.; Mayawala, K.; Poulin, P. 2012. Dose Selection Based on Physiologically Based Pharmacokinetic (PBPK) Approaches. The American Association of Pharmaceutical Scientists Journal, 15(2):377-387.

Leblanc, P, H. 1991. Chemical restraint for surgery in the standing horse. Veterinary Clinics of North America: Equine Practice. 7(3):521-533.

Love, E. J., Taylor, P. M., Murrell, J. et al. 2012. Effects of acepromazine, butorphanol and buprenorphine on thermal and mechanical nociceptive thresholds in horses. Equine Veterinary Journal, 44:221-225.

Love, E. J., LudovicPelligand, L., Polly, M., Taylor, T. M., Joanna, C., Murrell, J. C., John, W. \& Sear, J.W. 2015. Pharmacokineticpharmacodynamic modelling of intravenous buprenorphine in conscious horses Veterinary Anaesthesia and Analgesia, 42:17-29

Mama, K, R., Grimsrud, K., Snell, T. \& Stanley, S. 2009. Plasma concentrations, behavioral and physiological effects following intravenous and intramuscular detomidine in horses. Equine Veterinary Journal, 41(8)772-777.

Motta, E., Luglio, M., Delgado, A. F. \& Carvalho, W. B. 2016. Importância do uso de protocolos 
para manejo da analgesia e sedação em unidade de terapia intensiva pediátrica. Revista da Associação de Medicina Brasileira, 62:6.

Rohrbach, H., Korpivaara, T., Schatzmann, U. et al. 2009. Comparison of the effects of the alpha-2 agonist detomidine, romifidine and xylazine on nociceptive withdrawal reflex and temporal summation in horses.Veterinary Anaesthesia and Analgesia. 36(4):384-95.

Salonen, J. S., Vaha-vahe, T., Vainio, O. \& Vakkuri $1989 . \quad$ O. Single-dose pharmacokinetics of detomidine in the horse and cow. Journal of Veterinary Pharmacology and Therapeutics, 12:65-72.

Upton, R, N. \& Mould, D, R. 2014. Basic concepts in population modeling, simulation, and model-based drug development: part 3introduction to pharmacodynamic modeling methods. CPT Pharmacometrics System Pharmacological, 3(1):e88.
Valverde, A. 2010. Alpha-2 agonist as paintherapy in horse. Veterinary Clinics of North America: Equine Practice. 26(3):51532.

Virtanen R. 1986. Pharmacology of detomidine and other $\alpha 2$ adrenoceptor agonists in the brain. Acta Veterinary Scandinavian, Suppl. 82:3546.

Whiteside, G. T., Pomonis, J, D. \& Kennedy, J. D. 2013. An Industry perspective on the role and utility of animal models of pain in drug discovery. Neuroscience Letters. 557(A):6572 .

Recebido: 2 Outubro 2018.

Aprovado:9 Novembro 2018.

Publicado: 7 Dezembro 2018.

Licenciamento: Este artigo é publicado na modalidade Acesso Aberto sob a licença Creative Commons Atribuição 4.0 (CC-BY 4.0), a qual permite uso irrestrito, distribuição, reprodução em qualquer meio, desde que o autor e a fonte sejam devidamente creditados 